\title{
Cardioprotective activity of placental growth factor combined with oral supplementation of L-arginine in a rat model of acute myocardial infarction
}

Liyun Luol,*

Bairong Chen ${ }^{1, *}$

Yin Huang'

Zibin Liang ${ }^{2}$

Songbiao $\mathrm{Li}^{\prime}$

Yuelan Yin'

Jian Chen'

Wei Wu'

'Department of Cardiology,

${ }^{2}$ Department of Oncological

Radiotherapy, The Fifth Affiliated

Hospital of Sun Yat-Sen University,

Zhuhai, Guangdong, People's Republic

of China

*These authors contributed equally to this work
This article was published in the following Dove Press journal:

Drug Design, Development and Therapy

28 October 2016

Number of times this article has been viewed

Objective: Exogenous administration of placental growth factor (PlGF) stimulates angiogenesis and improves ventricular remodeling after acute myocardial infarction (AMI), and supplementation with L-arginine ameliorates endothelial function. The objective of the present study was to compare the cardioprotective effects of combination therapy of PIGF and L-arginine with those of direct administration of PlGF alone in a rat model of AMI.

Materials and methods: Fifty male Sprague Dawley rats were randomly divided into five groups: sham group, normal saline group, L-arginine group, PlGF group, and combination group (PlGF + L-arginine). An AMI rat model was established by ligation of the left anterior descending of coronary arteries. After 4 weeks of postligation treatment, cardiac function, scar area, angiogenesis and arteriogenesis, myocardial endothelial nitric oxide synthase (eNOS) and collagen I protein content, and plasma concentration of brain natriuretic peptide (BNP) were studied. Echocardiography, Masson's staining, immunohistochemical analyses, Western blot, and enzyme-linked immunosorbent assay were performed.

Results: Left ventricular ejection fraction (LVEF), left ventricular fraction shortening (LVFS), and capillary and arteriole densities were higher in the PlGF group than in the normal saline group $(P<0.01)$. Scar area, collagen I protein content, and plasma concentration of BNP were decreased in the PlGF group $(P<0.01)$. Myocardial eNOS protein level was elevated in the L-arginine group and PlGF $+\mathrm{L}$-arginine group $(P<0.01)$. Compared with the PlGF group, LVEF, LVFS, myocardial eNOS, and capillary and arteriole densities were higher in the combination group $(P<0.01)$. Scar area, content of collagen I protein, and plasma concentration of BNP were reduced in the combination group $(P<0.01)$.

Conclusion: Exogenous administration of PlGF stimulates angiogenesis and improves cardiac function. L-arginine increases the expression of the eNOS protein. PlGF and L-arginine have a more pronounced, synergistic protective effect on myocardial protection compared with that of exogenous PIGF therapy alone.

Keywords: placental growth factor, L-arginine, acute myocardial infarction, angiogenesis

\section{Introduction}

Acute myocardial infarction (AMI) is a serious threat to human life. It results in scar formation with remodeling and ventricular dilatation and ultimately leads to heart failure and death. Reperfusion of the occluded coronary artery is the most important way to save the ischemic myocardium and life. Although the reperfusion therapy can considerably reduce the mortality rate of AMI, the level of rehospitalization because of ischemic cardiomyopathy due to AMI is still high. ${ }^{1}$ Therapeutic angiogenesis is cc) ${ }_{\mathrm{BY}} \mathrm{NC}$ and incorporate the Creative Commons Attribution - Non Commercial (unported, v3.0) License (http://creativecommons.org/licenses/by-n/3.0/). By accessing the work you hereby accept the Terms. Non-commercial uses of the work are permitted without any further permission from Dove Medical Press Limited, provided the work is properly attributed. For permission for commercial use of this work, please see paragraphs 4.2 and 5 of our Terms (https://www.dovepress.com/terms.php). 
an attractive alternative approach to the treatment of myocardial ischemia, in which the induction of angiogenesis is used to preserve cardiac function by promoting the growth of new blood vessels and facilitating the production of new cardiomyocytes of the ischemic region. ${ }^{2,3}$ One of the paracrine factors is placental growth factor (PlGF). It is a $50-\mathrm{kDa}$ homodimeric glycoprotein sharing 53\% sequence homology at the amino acid level with the vascular endothelial growth factor (VEGF). ${ }^{4}$ Previous studies showed that VEGF did not reduce infarction size and improved cardiac function despite its strong angiogenic characteristics, while PlGF significantly improved ventricular remodeling by stimulating angiogenesis and arteriogenesis simultaneously, but without any side effects, such as edema, hypotension, and the formation of hemangiomas..$^{5-7}$

Nitric oxide (NO) is an important medium involved in a wide variety of regulatory mechanisms of the cardiovascular system, which is formed from the amino acid precursor L-arginine by the enzyme endothelial nitric oxide synthase (eNOS). Decrease of eNOS expression in ischemic myocardium leads to the reduction of NO synthesis and eventually causes endothelial dysfunction and a reduction of myocardial perfusion. Since L-arginine is a substrate for eNOS, its supplementation in animals improves endothelial function. ${ }^{8}$ This amino acid can also diminish the endothelial cell damage caused by various factors under hypoxic conditions and stimulate angiogenesis. ${ }^{9}$ However, the therapeutic effects of intramyocardial injection of PlGF combined with oral supplementation of L-arginine have not been investigated. In this study, it was hypothesized that the combination therapy provides a better cardioprotective activity in comparison with direct administration of PIGF in a rat model of AMI.

\section{Materials and methods}

\section{Animals}

Male Sprague Dawley rats (weighing 250 \pm 50 g, 8-10 weeks old) were obtained from the Experimental Animal Center, Sun Yat-Sen University, People's Republic of China. All animals received humane care in accordance with the guidelines issued by the National Science and Technology Commission of China. This experiment was approved by the The Fifth Affiliated Hospital of Sun Yat-Sen University ethics committee. Rats were housed in groups of three per cage and fed with free access to a formula diet and clean water, at $20^{\circ} \mathrm{C}-24^{\circ} \mathrm{C}$.

\section{Ligation of the left coronary artery and treatment}

The surgery of coronary artery ligation was performed as reported earlier. ${ }^{10,11}$ Rats were anesthetized using 1\% pentobarbital administered by intraperitoneal injection (30-45 mg/kg), under conditions of mechanical ventilation at $65-80$ breaths/minute after intubation and a tidal volume of $30 \mathrm{~mL} / \mathrm{kg}$. Thoracotomy was performed through the fourth/fifth intercostal space. The left anterior descending coronary artery was ligated with $6 / 0$ prolene suture, below the edge of the left atrial appendage $(2-3 \mathrm{~mm})$. Few minutes later, pallor was observed in the anterior wall corresponding to the distribution of the left anterior descending coronary artery territory. Appearance of a pale area and a huge R wave with a notch in electrocardiography monitoring was considered as a criteria for achieving a successful model of AMI. Rats were randomized into five groups. In sham operation group, $\mathrm{A}(\mathrm{n}=10)$, the coronary artery of the animals was not ligated after thoracotomy. The experimental rats in the control group $B(n=10)$ received intramyocardial injection of $300 \mu \mathrm{L}$ normal saline and those in the treatment group $\mathrm{C}$ $(\mathrm{n}=10)$ were given L-arginine (Sigma-Aldrich Co., St Louis, MO, USA) by gavage once a day $(100 \mathrm{mg} / \mathrm{kg})$ for 4 weeks. After the surgery, the animals in treatment group D $(n=10)$ received intramyocardial injection of $2 \mu \mathrm{g}$ of recombinant human PlGF-2 (rhPlGF-2) in $300 \mu \mathrm{L}$ of saline in the border zone. rhPlGF-2 was purchased from R\&D Systems, Inc. (Minneapolis, MN, USA). The rats in the treatment group E $(n=10)$ were administered an intramyocardial injection of $2 \mu \mathrm{g}$ of rhPlGF- 2 in $300 \mu \mathrm{L}$ of saline in the border zone after ligation; in addition, L-arginine was given by gavage once a day $(100 \mathrm{mg} / \mathrm{kg})$ for 4 weeks. Ten minutes after the ligation of the coronary artery, three intramyocardial injections were given precisely in the infarction border zone using a microsyringe (insulin pen).

\section{Cardiac function examinations}

Echocardiography examinations were performed under anesthesia using $1 \%$ pentobarbital by intraperitoneal injection. Transthoracic echocardiography was performed before the surgery (baseline data) and 4 weeks after ligation with the Philips ultrasound system (Philips iE33; Philips, Eindhoven, the Netherlands), equipped with a 12-MHz linear probe. The scanning speed was $150 \mathrm{~mm} / \mathrm{s}$. The left ventricular end-diastolic dimension (LVEDD) and left ventricular endsystolic dimension (LVESD) were measured. According to the obtained data, left ventricular end-diastolic volume (LVEDV), left ventricular end-systolic volume (LVESV), left ventricular ejection fraction (LVEF), and left ventricular fraction shortening (LVFS) were calculated using the Teichholz formula: ${ }^{12}$

$$
\mathrm{LVESV}=\left(7.0 \times \mathrm{LVESD}^{3}\right) /(2.4+\mathrm{LVESD})
$$




\section{$\mathrm{LVEDV}=\left(7.0 \times \mathrm{LVEDD}^{3}\right) /(2.4+\mathrm{LVEDD})$ \\ LVEF $=($ LVEDV - LVESV $) /$ LVEDV \\ LVFS $=($ LVEDD - LVESD $) /$ LVEDD}

\section{Scar area analysis}

Four weeks after coronary ligation, the rats were anesthetized by pentobarbital, and their hearts were excised, washed with cold saline to remove the residual blood from the ventricles, and fixed in $4 \%$ formalin. After embedding in paraffin, the ventricles were sectioned into $5-\mu \mathrm{m}$ transverse slices. In order to evaluate the infarct size, Masson's trichrome staining was performed, and the stained sections were captured as images and photographed using a microscope (CCD TP510; Optec, Inc., Lowell, MI, USA). The areas of fibrin deposition and those of viable tissue were measured, and the ratio of fibrin deposition area/viable tissue area was analyzed by Image-Pro Plus software. ${ }^{13}$

\section{Immunohistochemical analysis}

The rat hearts were fixed and embedded in frozen section medium. Immunohistochemical methods were applied to evaluate neovascularization and capillary and arteriole densities of the peri-infarct area. For this purpose, the following antibodies were used: anti-CD31 antibody (catalog no Ab5694; Abcam, Cambridge, MA, USA) for identification of endothelial cells and anti- $\alpha$ smooth muscle actin antibody (NB100-2284; Novus Biological, Littleton, CO, USA) for identification of smooth muscle cells. Briefly, five fields were selected and imaged in the peri-infarct zone, and the mean total CD31-positive microvessels and total smooth muscle alpha actin-positive microvessels were counted to represent capillary density and arteriole density. ${ }^{14}$

\section{Western blot}

Tissue specimens were collected from the infarct border zone, and $300 \mu \mathrm{L}$ of radioimmunoprecipitation assay was added to the mashed tissue after centrifugation. After their homogenization for 5-8 minutes, the intermediate layer of liquid was collected following repeated cycles of centrifugation. Aliquots of $20 \mu \mathrm{L}$ of whole-cell lysates from each sample prepared in a buffer containing $2 \%$ sodium dodecyl sulfate (SDS) were separated by electrophoresis on an SDSpolyacrylamide gel electrophoresis gel. Then, they were transferred into a polyvinylidene fluoride membrane blot.

After blocking with 5\% skim milk, the blot was incubated with primary antibodies against collagen I (catalog no ab34710, 1:3000 dilution; Abcam) and eNOS (catalog no ab66127, 1:1000 dilution; Abcam) at $4{ }^{\circ} \mathrm{C}$ overnight. The blot was washed twice every 7 minutes using Tris-buffered saline with tween (TBST). Next, it was incubated with a horseradish peroxidase anti-rabbit secondary antibody (No FSA003; Forevergen, Guangzhou, People's Republic of China) for 1-2 hours at room temperature. Electrogenerated chemiluminescence was developed after another round of washing with TBST. ${ }^{15}$ An analysis of the optical density value of the target band was conducted by the ImageJ software.

\section{Enzyme-linked immunosorbent assay (ELISA)}

Blood samples were collected from the abdominal aorta before the rats were euthanized. The blood serum was separated, and the levels of plasma concentration of brain natriuretic peptide (BNP) were measured by ELISA according to the manufacturer's instructions (CBS-E07972R; CUSABIO, Wuhan, Hubei, People's Republic of China). ${ }^{16}$

\section{Statistical analysis}

All results were expressed as mean \pm standard deviation. The differences among different groups were evaluated for statistical significance using one-way analysis of variance (ANOVA; normal distribution data) or Kruskal-Wallis test (non-normal distribution data). A probability value, $P<0.05$, was considered statistically significant. Statistical analyses were performed using SPSS Statistics 21.0 software (IBM Corporation, Armonk, NY, USA).

\section{Results \\ Echocardiography findings}

Echocardiography was performed before the surgery and 4 weeks after ligation. Before the surgery, there were no differences among the five groups in the values of LVEDD, LVESD, and LVEF $(P>0.05)$. As established through ANOVA, there were significant differences among the five groups in the levels of LVEDD, LVESD, LVEF, and LVFS after 4 weeks of ligation. Post hoc comparisons revealed that values of LVEDD, LVESD, LVEF, and LVFS in group B were not statistically significant compared with those in group $\mathrm{C}(P>0.05)$. The values of LVEDD and LVESD after 4 weeks of ligation in group B and group C were higher than those in group $\mathrm{D}$ and group $\mathrm{E}$, while the values of LVEF and LVFS in group D and group $\mathrm{E}$ were higher than those in group $\mathrm{B}$ and group $\mathrm{C}(P<0.01)$. Four weeks after the ligation, LVEDD and LVESD rates in group $\mathrm{D}$ were higher than those in group E, while the LVEF and LVFS values in group $\mathrm{E}$ were higher than those in group D $(P<0.01$; Figure 1$)$. 


\section{Scar area measurements}

The Masson's trichrome staining results are presented in Figure 2. Viable myocardium areas were stained red, whereas those with fibrosis were stained blue. No area was stained blue in the sham operation group. There was a significant difference among the five groups in the average scar percentage after 4 weeks of ligation. Post hoc comparisons showed that there was no significant difference between the mean scar percentage in group B $(36.01 \% \pm 2.40 \%)$ and group C $(35.57 \% \pm 0.95 \% ; P>0.05)$. However, the average scar percentages 4 weeks post infarction in group $\mathrm{B}$ and group $\mathrm{C}$ were higher than those in group D $(23.11 \% \pm 3.17 \%)$ and group $\mathrm{E}$
$(10.40 \% \pm 1.88 \% ; P<0.01)$, and the average scar percentage in group $\mathrm{E}$ was smaller than that in group $\mathrm{D}(P<0.01)$.

\section{Angiogenesis and arteriogenesis measurements}

In order to clarify the mechanism of the potential beneficial effects of PlGF and/or L-arginine in postinfarct pathology, angiogenesis and arteriogenesis in peri-infarct myocardium were analyzed by the immunohistochemical method. ANOVA manifested that there were significant differences among the five groups in the mean values of capillary density (CD31-positive microvessels $/ \mathrm{mm}^{2}$ ) 4 weeks post infarction.
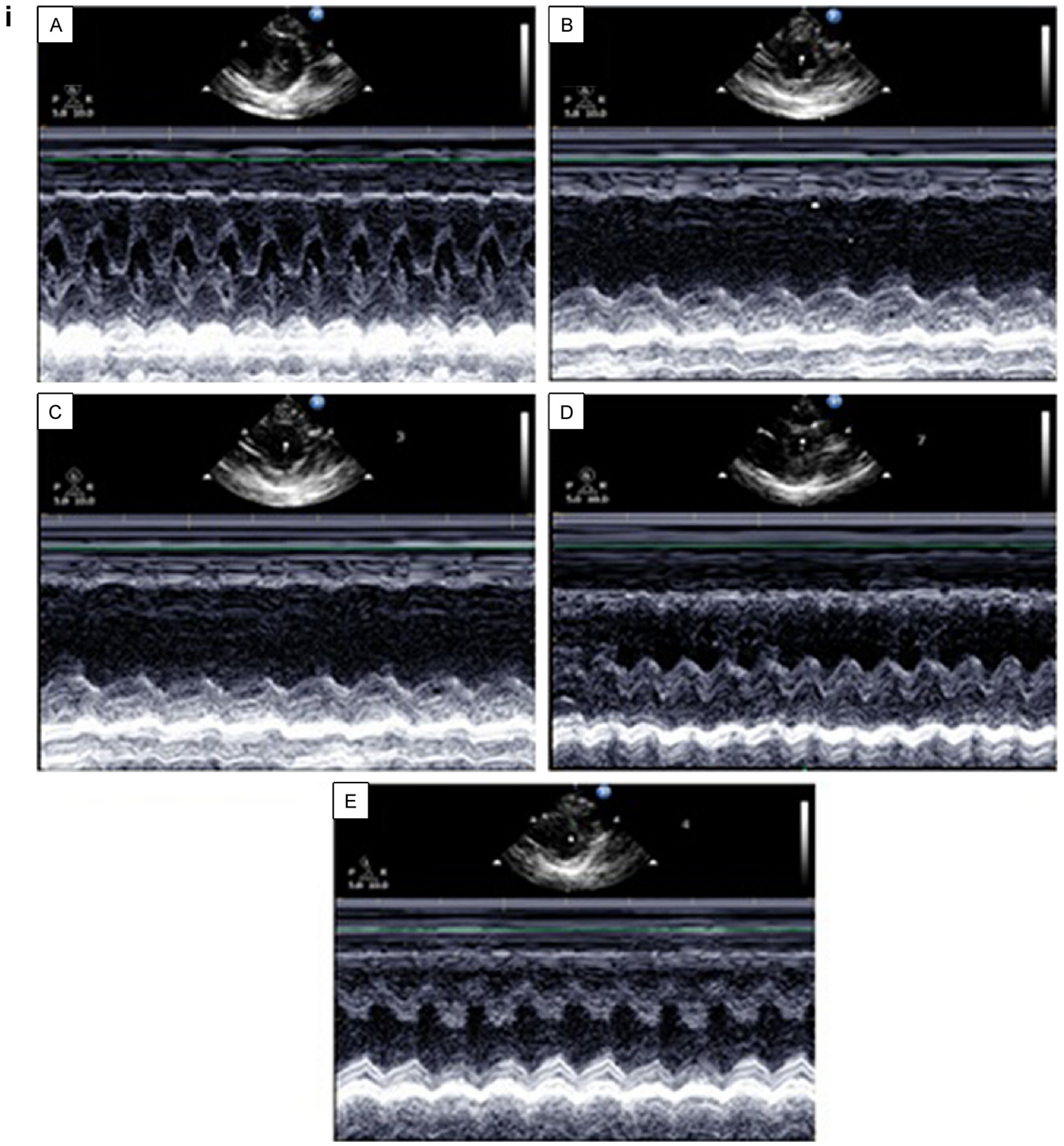

Figure I (Continued) 

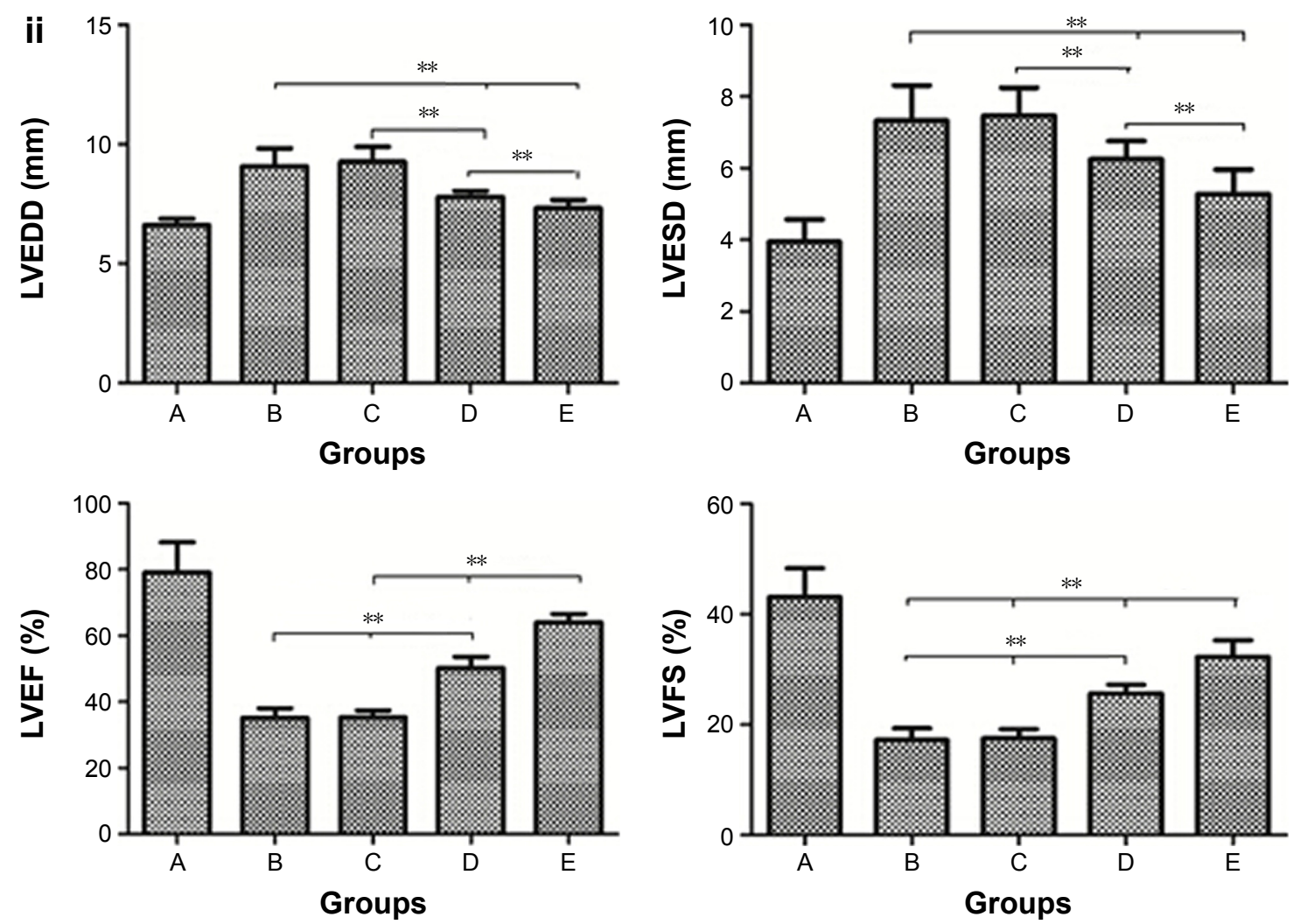

Figure I (i and ii) Echocardiography after 4 weeks of AMI.

Notes: Images of a heart from the sham group (A), injection with normal saline (B), treatment with L-arginine (C), intramyocardial injection with $2 \mu \mathrm{g}$ PIGF (D), and intramyocardial injection with $2 \mu \mathrm{g}$ PIGF and combined treatment of L-arginine (E). **P $<0.01$.

Abbreviations: AMI, acute myocardial infarction; PIGF, placental growth factor; LVEDD, left ventricular end-diastolic dimension; LVESD, left ventricular end-systolic dimension; LVEF, left ventricular ejection fraction; LVFS, left ventricular fraction shortening.
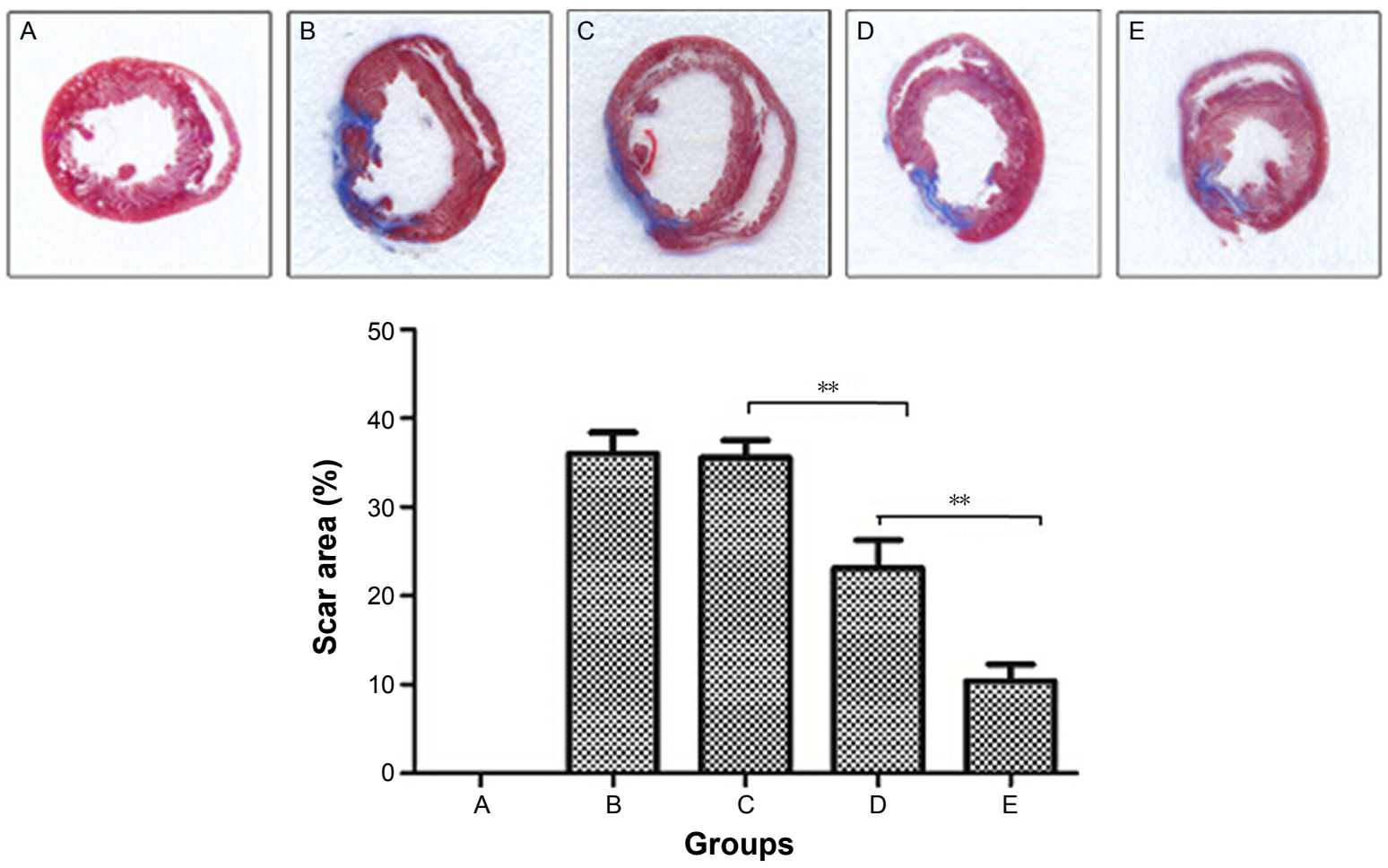

Figure 2 Myocardial scar formations after AMI.

Notes: Myocardial scar formations from the sham group (A), injection with normal saline (B), treatment with L-arginine (C), intramyocardial injection with $2 \mu \mathrm{g}$ PIGF (D), and intramyocardial injection with $2 \mu \mathrm{g}$ PIGF and combined treatment with L-arginine (E). Viable myocardium was stained red, and fibrosis was stained blue. $* * P<0.0 \mathrm{I}$. Abbreviations: AMI, acute myocardial infarction; PIGF, placental growth factor. 
Post hoc comparisons indicated that there was significant difference in the mean capillary density between group B $\left(34.8 \pm 5.15 / \mathrm{mm}^{2}\right)$ and group $\mathrm{C}\left(48.8 \pm 4.53 / \mathrm{mm}^{2} ; P<0.05\right)$. As depicted in Figure 3, the levels of the mean capillary density in group $\mathrm{D}\left(131.4 \pm 7.74 / \mathrm{mm}^{2}\right)$ and group $\mathrm{E}\left(150.2 \pm 5.34 / \mathrm{mm}^{2}\right)$ were higher than those in group $\mathrm{B}$ and group $\mathrm{C}(P<0.01)$; the mean capillary density in group $\mathrm{E}$ was higher than that in group $\mathrm{D}(P<0.01)$.

Similarly, ANOVA analysis manifested that there was a significant difference among the five experimental groups in average arteriole density 4 weeks post infarction. Post hoc comparisons exhibited no significant difference between group B $\left(7.2 \pm 1.72 / \mathrm{mm}^{2}\right)$ and group C $\left(10.8 \pm 2.14 / \mathrm{mm}^{2}\right)$ $(P>0.05)$, while the values of the average arteriole density in group D $\left(24.2 \pm 3.71 / \mathrm{mm}^{2}\right)$ and group $\mathrm{E}\left(35.2 \pm 3.12 / \mathrm{mm}^{2}\right)$ were higher than those in group $\mathrm{B}$ and group $\mathrm{C}(P<0.01)$. As illustrated in Figure 4, the average arteriole density in group $\mathrm{E}$ was higher than that in group $\mathrm{D}(P<0.01)$.

\section{Western blot analysis}

The results of the densitometry analysis of protein levels in the peri-infarct area are displayed in Figure 5. Myocardial eNOS and collagen I protein content were significantly different among the five groups. Myocardial eNOS content in group B and group D was lower than that in group C and group $\mathrm{E}(P<0.01)$, whereas no significant difference was found between group $\mathrm{B}$ and group $\mathrm{D}(P>0.05)$ and between group $\mathrm{C}$ and group $\mathrm{E}(P>0.05)$. The levels of collagen I protein in group $\mathrm{B}$ and group $\mathrm{C}$ were higher than those in group $\mathrm{D}$ and group $\mathrm{E}(P<0.01)$, whereas there was no significant difference in these levels between group $\mathrm{B}$ and group $\mathrm{C}$ $(P>0.05)$ and between group $\mathrm{D}$ and group $\mathrm{E}(P>0.05)$.

\section{Plasma concentration of BNP}

ANOVA results manifested that the plasma BNP level was significantly different among the five groups. Post hoc comparisons showed that there was no significant difference
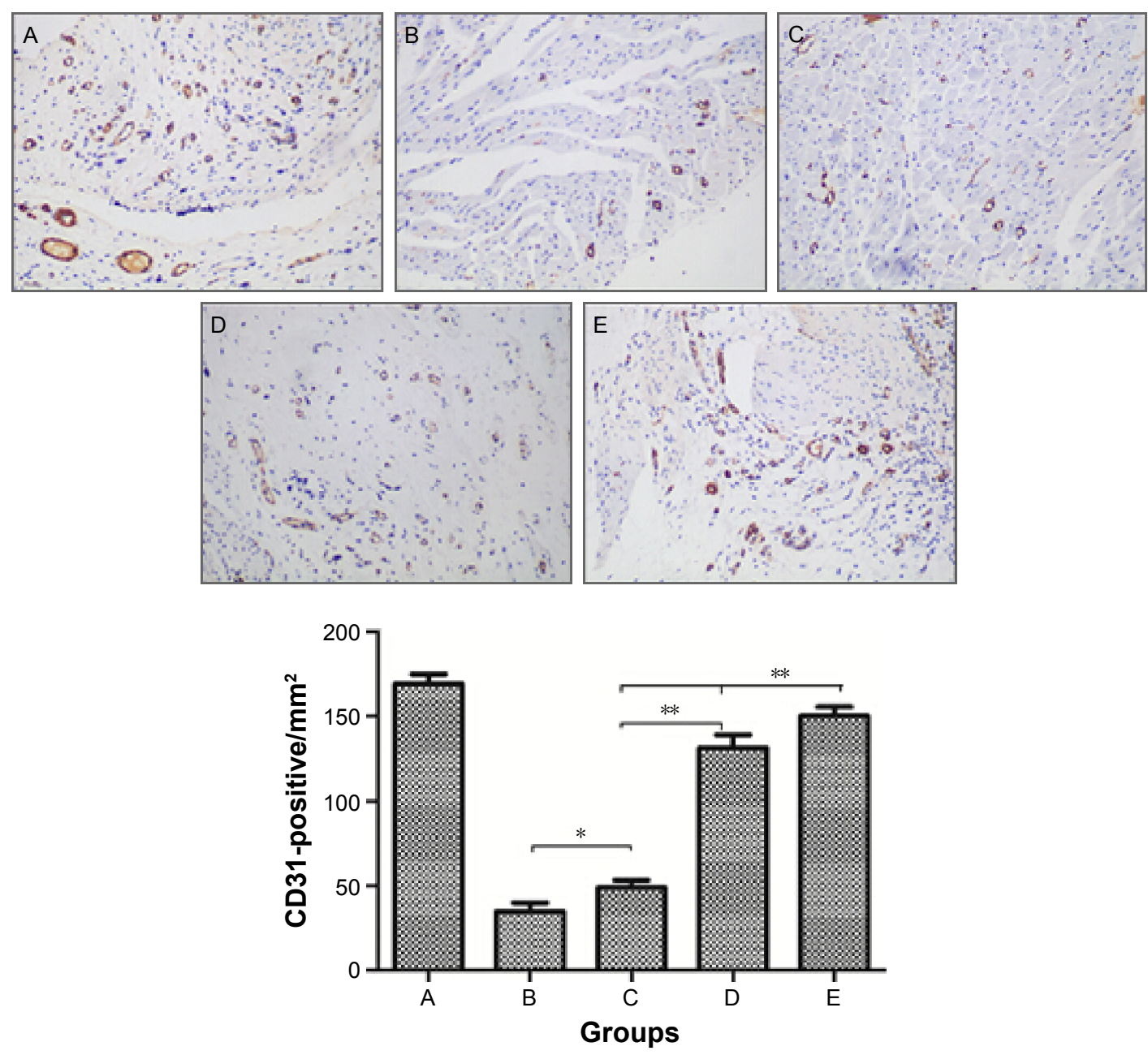

Figure 3 Angiogenesis in the peri-infarct area.

Notes: $* P<0.05$. $* * P<0.01$. A, sham group; B, injection with normal saline; C, treatment with L-arginine; D, intramyocardial injection with PIGF; E, intramyocardial injection with $2 \mu \mathrm{g}$ PIGF and combined treatment of L-arginine. Magnification $\times 200$.

Abbreviation: PIGF, placental growth factor. 

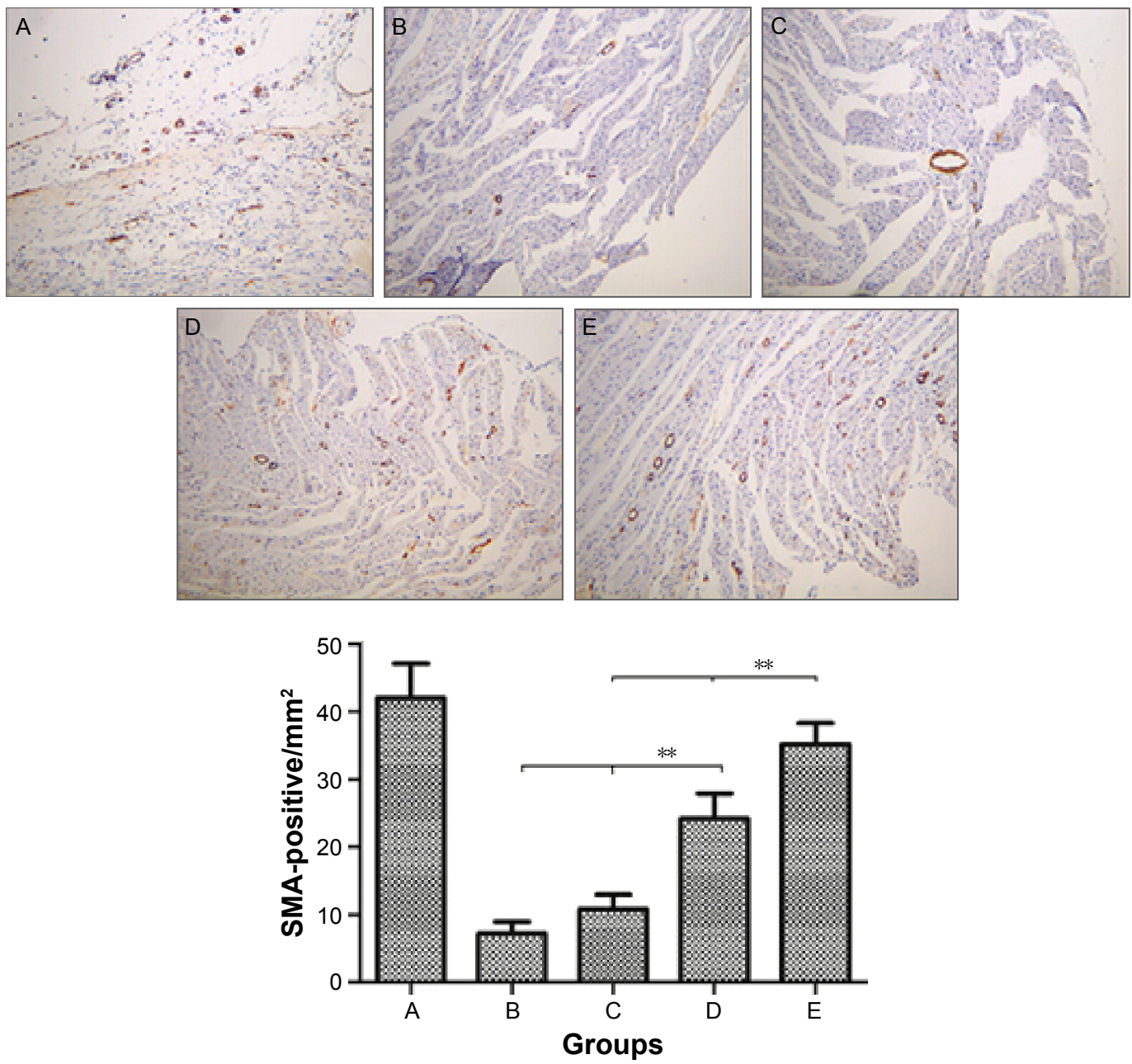

Figure 4 Arteriogenesis in the peri-infarct area.

Notes: $* * P<0.0 I$. A, sham group; B, injection with normal saline; C, treatment with L-arginine; $D$, intramyocardial injection with PIGF; E, intramyocardial injection with $2 \mu \mathrm{g}$ PIGF and combined treatment of L-arginine. Magnification $\times 100$.

Abbreviation: PIGF, placental growth factor.

between group B $(1,203 \pm 100.83 \mathrm{pg} / \mathrm{mL})$ and group $\mathrm{C}$ $(1,111 \pm 86.78 \mathrm{pg} / \mathrm{mL} ; P>0.05)$. The level of plasma BNP in group $\mathrm{C}(1,111 \pm 86.78 \mathrm{pg} / \mathrm{mL})$ was higher than that in group $\mathrm{D}$ $(672.33 \pm 48.88 \mathrm{pg} / \mathrm{mL})$ and group $\mathrm{E}(257 \pm 46.3 \mathrm{pg} / \mathrm{mL}$; $P<0.01)$, and the BNP level in group D was higher than that in group $\mathrm{E}(P<0.01 ;$ Figure 6$)$.

\section{Discussion}

Despite the popularity of reperfusion therapy and the fact that it saved many lives of patients with AMI, ischemic heart disease remains a serious threat to human life. Therapeutic angiogenesis is a novel approach to the treatment of AMI. Among numerous growth factors, PlGF has gradually become a hot area of research because of its strong dual function of promoting angiogenesis and arteriogenesis. As a member of the VEGF family, PIGF acts on different types of cells, endothelial cells, smooth muscle cells, inflammatory cells, and endothelial progenitor cells (EPCs), by binding with the fms-like tyrosine kinase receptor. ${ }^{17-19} \mathrm{PlGF}$ induces new vascular formation by two mechanisms: ${ }^{6,20}$ stimulation of the local proliferation of endothelial cells and smooth muscle cells and recruitment of EPCs to the myocardium tissue. Recent examinations have revealed that exogenous administration of rhPlGF improves cardiac function and enhances anti-inflammatory cytokine expression; it also reduces the infarction area and regulates metalloproteinasemediated myocardial tissue remodeling. ${ }^{5,21}$

Similar conclusions have been reached in the present study, in which the exogenous administration of rhPlGF-2 improved cardiac function (LVEF and LVFS), reduced infarction size, and promoted angiogenesis and arteriogenesis. A previous research focused on the tissue expression of $\mathrm{BNP} ;{ }^{6}$ for this reason, we decided to investigate the change in the serum level. The findings of this study evidenced that rhPlGF-2 therapy decreased plasma levels of BNP, a specific indicator reflecting the severity of heart failure. 

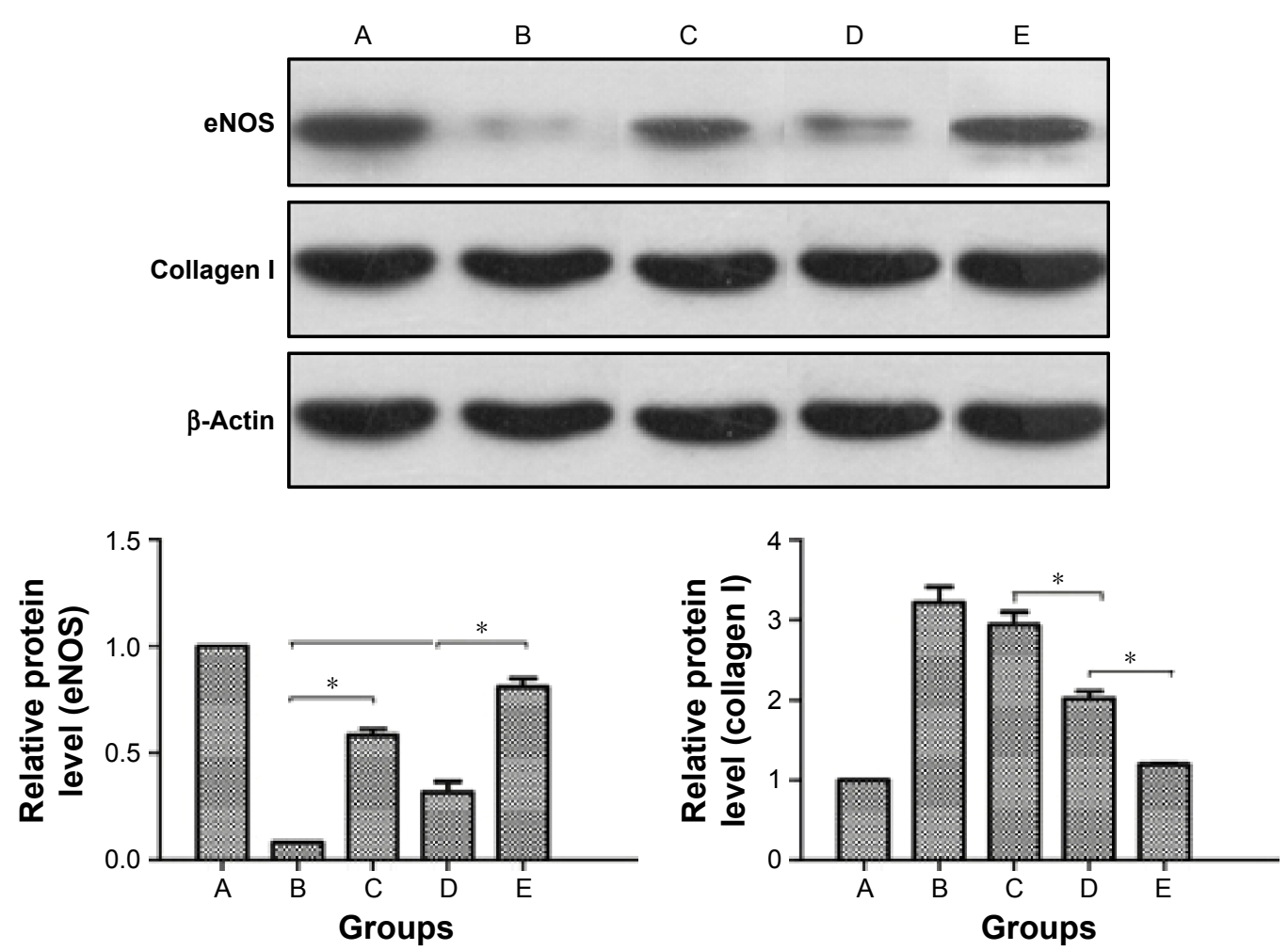

Figure 5 Content changes of eNOS, collagen I detected by Western blot.

Notes: Images of Western blot from the sham group (A), injection with normal saline (B), treatment with L-arginine (C), intramyocardial injection with $2 \mu \mathrm{g}$ PIGF (D), and intramyocardial injection with $2 \mu \mathrm{g}$ PIGF and combined treatment with L-arginine $(\mathrm{E})$. ${ }^{* P}<0.05$.

Abbreviations: eNOS, endothelial nitric oxide synthase; PIGF, placental growth factor.

In addition to the aforementioned beneficial effects, rhPlGF may also suppress collagen I deposition in the peri-infarct area. Collagen I is the main component of the intercellular matrix and plays an important role in the development of myocardial fibrosis after AMI. Thus, the regulation of collagen synthesis has become a crucial target for the treatment of left ventricular remodeling. ${ }^{22} \mathrm{PlGF}$ improves left ventricular remodeling and ultimately leads to the achievement of the aim of myocardial

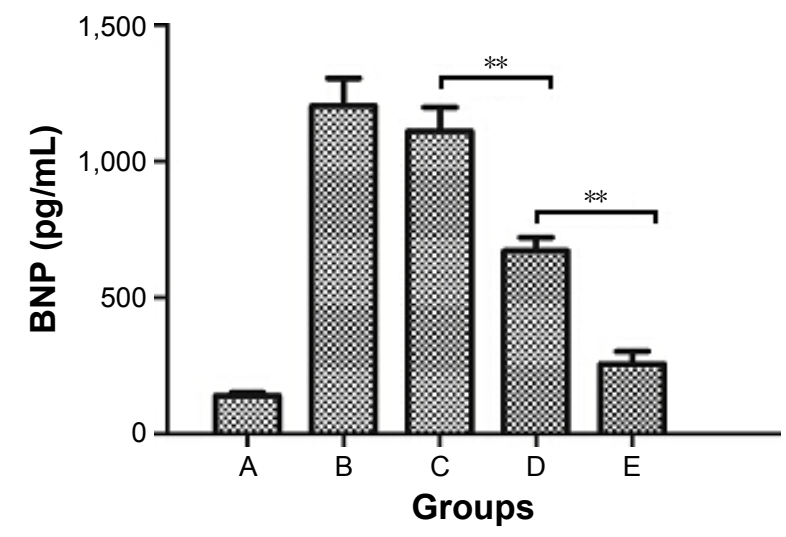

Figure 6 Plasma concentration of BNP by ELISA.

Notes: BNP of Sham group (A), injection with normal saline (B), treatment with L-arginine (C), intramyocardial injection with $2 \mu \mathrm{g}$ PIGF (D), and intramyocardial injection with $2 \mu \mathrm{g}$ PIGF and combined treatment with L-arginine (E). $* * P<0.01$.

Abbreviations: BNP, brain natriuretic peptide; ELISA, enzyme-linked immunosorbent assay; PIGF, placental growth factor. protection by reducing the expression of collagen I. However, the pathways through which rhPlGF treatment suppresses the deposition of collagen I in the peri-infarct area in the chronic phase remain unknown. The first feasible mechanism might be the alleviation of tissue hypoxia caused by new vascular formation. After rhPlGF intervention and preservation of blood supply, the proportions of both tissue inhibitors, TIMP metallopeptidase inhibitor 2 and matrix metalloproteinase-2 (MMP-2), increase in the total protein expression, resulting in acceleration of collagen I degradation. ${ }^{21}$ Another possibility is that inflammatory cells and factors play an important role in the pathophysiology of myocardial fibrosis. Inflammatory cells are recruited and activated in the ischemic area after AMI and secrete different kinds of cytokines, chemotactic factors, and adhesion molecules. Subsequently, fibroblasts are activated by these factors and synthesize collagen. The results of a previous study evidenced that PlGF exerted an effect on inflammatory cell activities and reduced the release of inflammatory factors, such as tumor necrosis factor- $\alpha$ and interleukin 6, which have a negative impact on the infarcted myocardium by causing remodeling. ${ }^{5}$

Previous evidence has not clarified the cardioprotective mechanisms of PlGF, and the potential techniques to optimize its protective effects deserve further exploration. Previous 
studies have focused on dosage, approaches, or time of administration, whereas few of them have concentrated on improving endothelial environment. ${ }^{5,6,7,21,23}$

To the best of our knowledge, we investigated for the first time the effects of the combined therapy of PlGF and L-arginine. Endothelial dysfunction in the hypoxic myocardial tissue is often severe, and poor endothelial environment reduces the treatment response of cytokines promoting angiogenesis. ${ }^{24}$ This study found that myocardial eNOS expression decreased in the chronic ischemia area, and oral supplementation of L-arginine ameliorated the expression of eNOS. Based on this rationale, we speculated that the combination therapy of rhPlGF and L-arginine might have a better myocardial protection effect. The findings of our examination confirmed that the combination treatment showed more advantages in improving cardiac function, reducing infarction area, promoting angiogenesis, and decreasing collagen-1 expression compared with exogenous rhPlGF therapy alone. Nevertheless, the application of L-arginine alone has not induced the effect of improved ventricular remodeling. According to our experimental results, rhPlGF and L-arginine have a synergistic effect in myocardial protection. The possible mechanism might be associated with the fact that PIGF depends heavily on the release of endothelial-derived $\mathrm{NO}^{3}$ and L-arginine improves endothelial function by ameliorating the expression of eNOS and NO, ultimately enhancing myocardial tissue perfusion. Another option could be that L-arginine can also reduce endothelial cell damage under hypoxic conditions, so as to maintain the stability of new blood vessels. ${ }^{9}$ The last but not the least, possibility might be that the level of asymmetric dimethylarginine, an arginine analog that competes with L-arginine for eNOS, is increased in AMI and can also lead to the impairment of endothelial function, and the competitive inhibition of eNOS by asymmetric dimethylarginine may be reversed by the supplementation of L-arginine. ${ }^{25}$

\section{Limitations}

However, this study has some limitations. For instance, coronary artery ligation of rats is difficult to perform, so it is laborious to achieve a high-quality and identical infarction area. This limitation is caused by the modeling method. In addition, study on the mechanism of neovascularization had not been delved deep enough.

\section{Conclusion}

Exogenous administration of PlGF stimulates angiogenesis and improves cardiac function. L-arginine increases the expression of the eNOS protein. PlGF and L-arginine have a more pronounced, synergistic protective effect on myocardial protection compared with that of exogenous PlGF therapy alone.

Although the prospect of therapeutic neovascularization is promising, its mechanisms are not completely elucidated, and a long period of exploration and transition to successful clinical trials will be needed. ${ }^{25}$ Modification and optimization of the vascular environment are crucial for effective neovascularization, and a multimodality approach requiring combinations of protein-, gene-, and cell-based therapies will be the focus of future research. ${ }^{26}$

\section{Disclosure}

The authors report no conflicts of interest in this work.

\section{References}

1. Singh P, Sethi N, Kaur N, Kozman H. Revascularization in severe left ventricular dysfunction: does myocardial viability even matter? Clin Med Insights Cardiol. 2015;9(Suppl 1):105-109.

2. Van der Laan AM, Piek JJ, van Royen N. Targeting angiogenesis to restore the microcirculation after reperfused MI. Nat Rev Cardiol. 2009; 6(8):515-523.

3. Lassaletta AD, Chu LM, Sellke FW. Therapeutic neovascularization for coronary disease: current state and future prospects. Basic Res Cardiol. 2011;106(6):897-909.

4. Nagy JA, Dvorak AM, Dvorak HF. VEGF-A (164/165) and PlGF: roles in angiogenesis and arteriogenesis. Trends Cardiovasc Med. 2003; 13(5):169-175.

5. Binsalamah ZM, Paul A, Khan AA, Prakash S, Shum-Tim D. Intramyocardial sustained delivery of placental growth factor using nanoparticles as a vehicle for delivery in the rat infarct model. Int J Nanomedicine. 2011;6:2667-2678.

6. Takeda Y, Uemura S, Iwama H, et al. Treatment with recombinant placental growth factor (PlGF) enhances both angiogenesis and arteriogenesis and improves survival after myocardial infarction. Circ J. 2009; 73(9):1674-1682.

7. Roncal C, Buysschaert I, Chorianopoulos E, et al. Beneficial effects of prolonged systemic administration of PIGF on late outcome of postischemic myocardial performance. J Pathol. 2008;216(2):236-244.

8. Böger RH, Ron ES. L-Arginine improves vascular function by overcoming deleterious effects of ADMA, a novel cardiovascular risk factor. Altern Med Rev. 2005;10(1):14-23.

9. Martínez-Romero R, Cañuelo A, Siles E, Oliver FJ, Martínez-Lara E. Nitric oxide modulates hypoxia-inducible factor-1 and poly (ADPribose) polymerase-1 cross talk in response to hypobaric hypoxia. J Appl Physiol. 2012;112(5):816-823.

10. Al Kindi AH, Asenjo JF, Ge Y, et al. Microencapsulation to reduce mechanical loss of microspheres: implications in myocardial cell therapy. Eur J Cardiothorac Surg. 2011;39(2):241-247.

11. Chen J, Zheng S, Huang H, et al. Mesenchymal stem cells enhanced cardiac nerve sprouting via nerve growth factor in a rat model of myocardial infarction. Curr Pharm Des. 2014;20(12):2023-2029.

12. Wykrzykowska JJ, Rosinberg A, Lee SU, et al. Autologous cardiomyotissue implantation promotes myocardial regeneration, decreases infarct size and improves left ventricular function. Circulation. 2011; 123(1):62-69.

13. Chen G, Nayan M, Duong M, et al. Marrow stromal cells for cell-based therapy: the role of anti-inflammatory cytokines in cellular cardiomyoplasty. Ann Thorac Surg. 2010;90(1):190-197.

14. Rendell MS, Finnegan MF, Pisarri T, et al. A comparison of the cutaneous microvascular properties of the spontaneously hypertensive rat and the Wistar-Kyoto rat. Comp Biochem Physiol A Mol Integr Physiol. 1999; 122(4):399-406. 
15. Voisine P, Bianchi C, Khan TA, et al. Normalization of coronary micro vascular reactivity and improvement in myocardial perfusion by surgical vascular endothelial growth factor combined with oral supplementation of L-arginine in a porcine model of endothelial dysfunction. J Thoral Cardiocasc Surg. 2005;129(6):1414-1420.

16. Li J, Yin FF, Hou YL. Early diagnosis of rats with acute myocardial infarction by measurement of brain natriuretic peptide. Exp Ther Med. 2013; 5(4):1201-1205.

17. Ziche M, Maglione D, Ribatti D, et al. Placenta growth factor-1 is chemotactic, mitogenic, and angiogenic. Lab Invest. 1997;76(4):517-531.

18. Bellik L, Cristina Vinci M, Filippi S, Ledda F, Parenti A. Intracellular pathways triggered by the selective FLT-1-agonist placental growth factor in vascular smooth muscle cells exposed to hypoxia. $\mathrm{Br} J$ Pharmacol. 2005;146(4):568-575.

19. Arras M, Ito WD, Scholz D, Winkler B, Schaper J, Schaper W. Monocyte activation in angiogenisis and collateral growth in the rabbit hindlimb. J Clin Invest. 1998;101(1):40-50.

20. Luttun A, Tjwa M, Moons L, et al. Revascularization of ischemic tissues by PIGF treatment, and inhibition of tumor angiogenesis arthritis and atherosclerosis by anti-Flt-1. Nat Med. 2002;8(8):831-840.

21. Lu ZX, Mao LL, Lian F, et al. Cardioprotective activity of placental growth factor in a rat model of acute myocardial infarction: nanoparticle-based delivery versus direct myocardial injection. BMC Cardiovasc Disord. 2014;14:53.
22. Bornstein P. Matricellular proteins: an overview. JCell Commun Signal. 2009;3(3-4):163-165.

23. Iwasaki H, Kawamoto A, Tjwa M, et al. PlGF repairs myocardial ischemia through mechanisms of angiogenesis, cardio protection and recruitment of myo-angiogenic competent marrow progenitors. PLoS One. 2011;6(9):e24872.

24. Riel M, Wu GF. Inhibition of the cardiac angiogenic response to surgical FGF-2 therapy in a swine endothelial dysfunction model. Circulation. 2003;108(suppl 1):335-340.

25. Walker HA, McGing E, Fisher I. Endothelium-dependent vasodilatation is independent of the plasma L-arginine/ADMA ratio in men with stable angina: lack of effect of oral L-arginine on endothelial function, oxidative stress and exercise performance. J Am Coll Cardiol. 2001; 38(2):499-505.

26. Antonio D, Lassaletta Louis M, Chu Frank W. Therapeutic neovascularization for coronary disease: current state and future prospects. Basic Res Cardiol. 2011;106(6):897-909.
Drug Design, Development and Therapy

\section{Publish your work in this journal}

Drug Design, Development and Therapy is an international, peerreviewed open-access journal that spans the spectrum of drug design and development through to clinical applications. Clinical outcomes, patient safety, and programs for the development and effective, safe, and sustained use of medicines are the features of the journal, which

\section{Dovepress}

has also been accepted for indexing on PubMed Central. The manuscript management system is completely online and includes a very quick and fair peer-review system, which is all easy to use. Visit http://www.dovepress.com/testimonials.php to read real quotes from published authors.

Submit your manuscript here: http://www.dovepress.com/drug-design-development-and-therapy-journal 